\title{
Periodontal plastic surgery techniques equally effective at root coverage
}

\author{
In people who have buccal gingival recession, is periodontal plastic surgery effective?
}

\begin{abstract}
Roccuzzo M, Bunino M, Needleman I, Sanz M. Periodontal plastic surgery for treatment of localized gingival recessions: a systematic review. J Clin Periodontol 2002; 29 (Suppl. 3):178-194

Data sources MEDLINE and the Cochrane Oral Health Group Trials Register were data sources; hand searches were made of reference lists from relevant articles, and of selected journals dated up to April 2001. Study selection Studies included were randomised and controlled trials, and case-series of at least 6 months' follow-up. The following interventions were assessed: coronally advanced flap (CAF), lateral positioned flaps, free gingival graft, connective tissue graft (CTG), guided tissue regeneration (GTR) with resorbable membranes and GTR with non-resorbable membranes.
\end{abstract}

Data extraction and synthesis Information regarding quality and study characteristics was extracted independently by two reviewers. Kappa scores determined their agreement. The following clinical outcomes were assessed: change in gingival recession; changes in clinical attachment gain; mean percentage of root coverage; and the proportion of sites with complete root coverage. In addition, long-term and patient-centred outcomes of long-term stability of root coverage (>12 months); and aesthetic satisfaction (unchanged, better, or worse, in the patient's opinion) were considered.

Results Thirty studies were eligible for inclusion. The results of this review showed a statistically significant reduction of gingival recession, with a concomitant attachment gain, following treatment with all the surgical techniques tested. A greater reduction was found by metaanalysis in recession following treatment with CTG than GTR (weighted mean difference, $0.43 \mathrm{~mm}$; 95\% confidence interval, $0.62-0.23$ ) and there was no statistically significant heterogeneity between the six studies included. For other comparisons, no differences were detected comparing CAF with GTR or resorbable versus non-resorbable membranes.

Conclusions Overall, periodontal plastic surgery was effective in reducing gingival recession with a concomitant improvement in attachment levels. Even though no single treatment can be considered superior to all the others, CTG was statistically significantly more effective than GTR in recession reduction. Further research is needed to identify the factors most associated with successful outcomes.

\section{Commentary}

Gingival recession is a clinical problem that dentists face on a daily basis. Patients have an increased risk of root caries, for which limited restorative options exist. Aesthetics may be compromised in people who with a high lip line and root exposure often causes dentinal hypersensitivity. This can significantly affect quality of life by limiting the types of foods patients can eat and sensitivity can interfere with normal hygiene procedures, leading to increased plaque accumulation and increased risk of further recession. Over half the adult population has at least one site of gingival recession, ${ }^{1}$ meaning there is a significant burden of illness as a result.

A number of periodontal "plastic surgery" procedures have been used to cover exposed roots: CTG, free gingival grafts, coronally positioned flaps, lateral sliding flaps, and the use of barrier membranes (GTR). The success of the procedure is dependent on a number of factors. These include the experience, the nature of the gingival defect, anatomical considerations and whether or not the patient smokes.

Address for correspondence: Dr Mario Roccuzzo, Corso Tassoni 14, 10144 Torino, Italy. E-mail: mroccuzzo@iol.it
This review is thorough, well-documented and transparent, key components of a good systematic review. However, the included studies only examined Miller's Class I and II defects. ${ }^{2}$ These are sites with no interproximal bone loss, and the ones most likely to result in $100 \%$ root coverage. It should be no surprise, therefore, that the results showed that, with the exception of free gingival grafts and lateral sliding flaps, most surgical techniques are successful at producing root coverage.

What is interesting is that CTG are slightly more effective than GTR membranes. Although a difference of $0.43 \mathrm{~mm}$ may not be clinically significant, one must take into account all the risks and benefits of these options. CTG have been shown to provide excellent root coverage, but the procedure necessitates the use of two sites a donor site (often the palate) and the recipient site. It is assumed that patients are more uncomfortable post-surgically as a result. With GTR, especially with bioresorbable membranes, there is only one surgical site. (Nonresorbable membranes require a second surgery to remove the membrane and thus offer no advantage over CTG.) Presumably, having one site would produce less postoperative morbidity. The most significant drawback to using GTR is cost, an important consideration in making choices. Given that most of the concerns about recession are related to quality-of-life, one would assume any clinical research would also focus on improvement in aesthetics (from the patient's point of view), changes in root sensitivity and cost-benefit. However, as the authors note such research is sadly lacking.

The paper reaches conclusions and makes recommendations for research and clinical practice. The latter two are important because the purpose of a systematic review is both to inform researchers about future directions and to inform clinicians of the most effective therapy in a given situation. To this end, I would recommend that the clinical recommendations of all reviews include a description of the patient population to which the recommendations apply. As well, use of a grading system ${ }^{3}$ would aid in determining the certainty with which one could apply the recommendations, based on the level of evidence.

\section{Practice point}

- With the exception of free gingival grafts and lateral sliding flaps, most surgical techniques are successful at producing root coverage for Miller's Class I and II defects.

\section{Debora Matthews}

Head, Division of Periodontics, Dalhousie University, Halifax, Nova Scotia, Canada

1. Kassab MM, Cohen RE. The etiology and prevalence of gingival recession. J Am Dent Assoc 2003; 134:220-225.

2. Miller Jr PD. A classification of marginal tissue recession. Int J Periodontol Rest Dent 1985; 18:444-453.

3. Woolf S, Battista R, Anderson G, Logan A, Wang E. Assessing the clinical effectiveness of preventive maneuvers: analytic principles and systematic methods in reviewing evidence and developing clinical practice recommendations. A report by the Canadian Task Force on the Periodic Health Examination. J Clin Epidemiol 1990; 43:891-905. 